\title{
On High-Performance Parallel Decimal Fixed-point Multiplier Designs
}

Ming Zhu

zhum2@unlv.nevada.edu

\section{Introduction}

Decimal computations are required in finance, and etc.

- Precise representation for decimals (E.g. 0.2, 0.7... )

- Performance Requirements (Software simulations are very slow)

Traditional Approach (8421 BCD)

$$
\begin{aligned}
& P_{2 n}=A_{n} \times B_{n} \\
& =A_{n}\left(\sum_{i=0}^{n-1} b_{i}(10)^{i}\right) \\
& =\sum_{i=0}^{n-1} A_{n} b_{i}(10)^{i} \\
& =\sum_{i=0}^{n-1} P P i_{n+1}(10)^{i}
\end{aligned}
$$

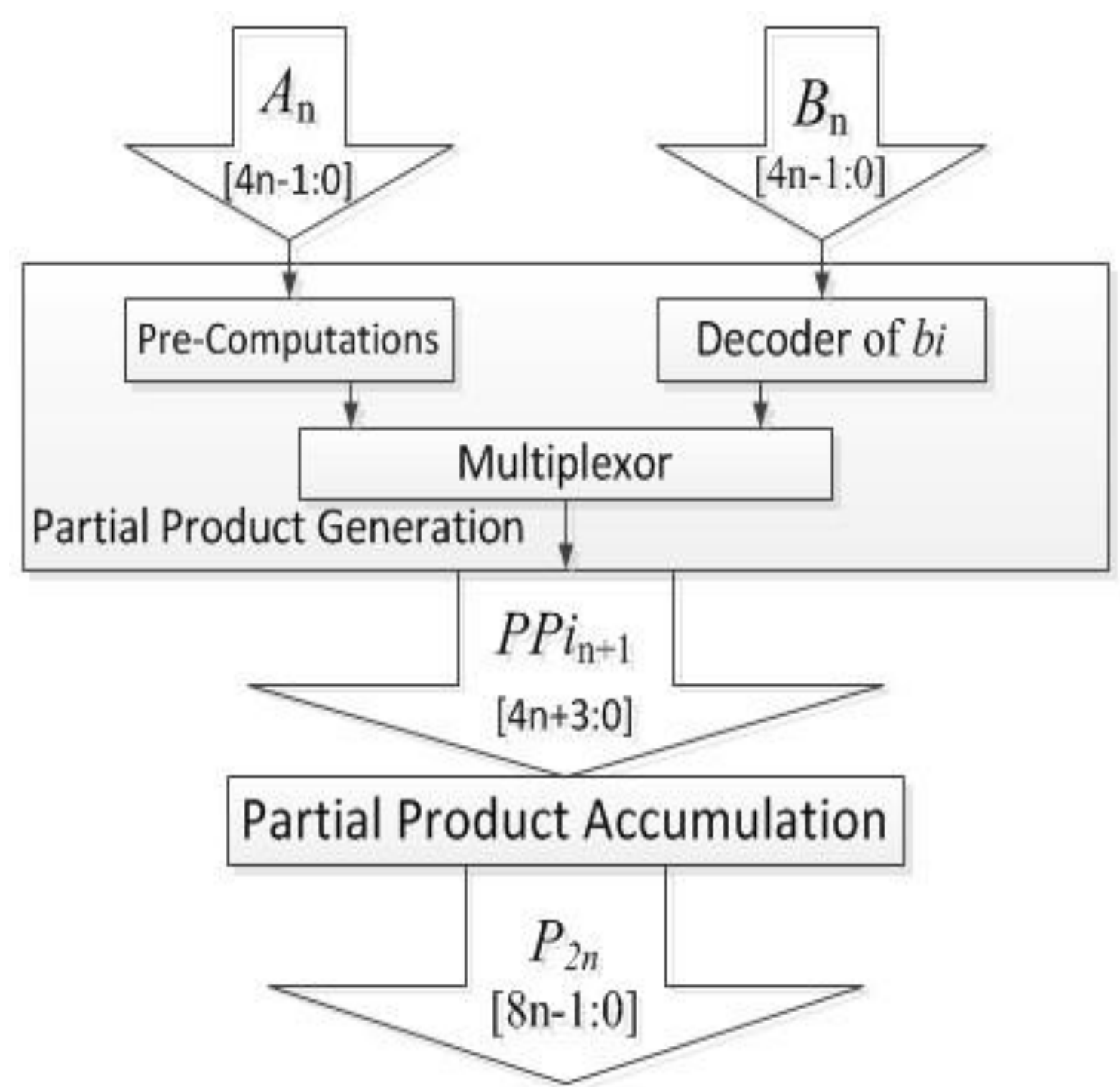

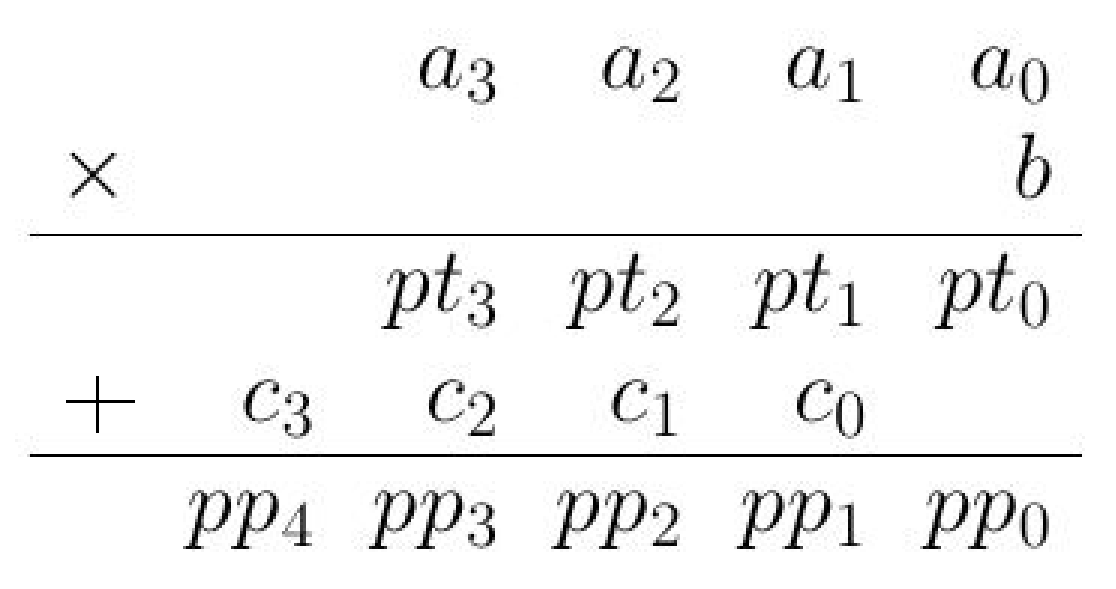

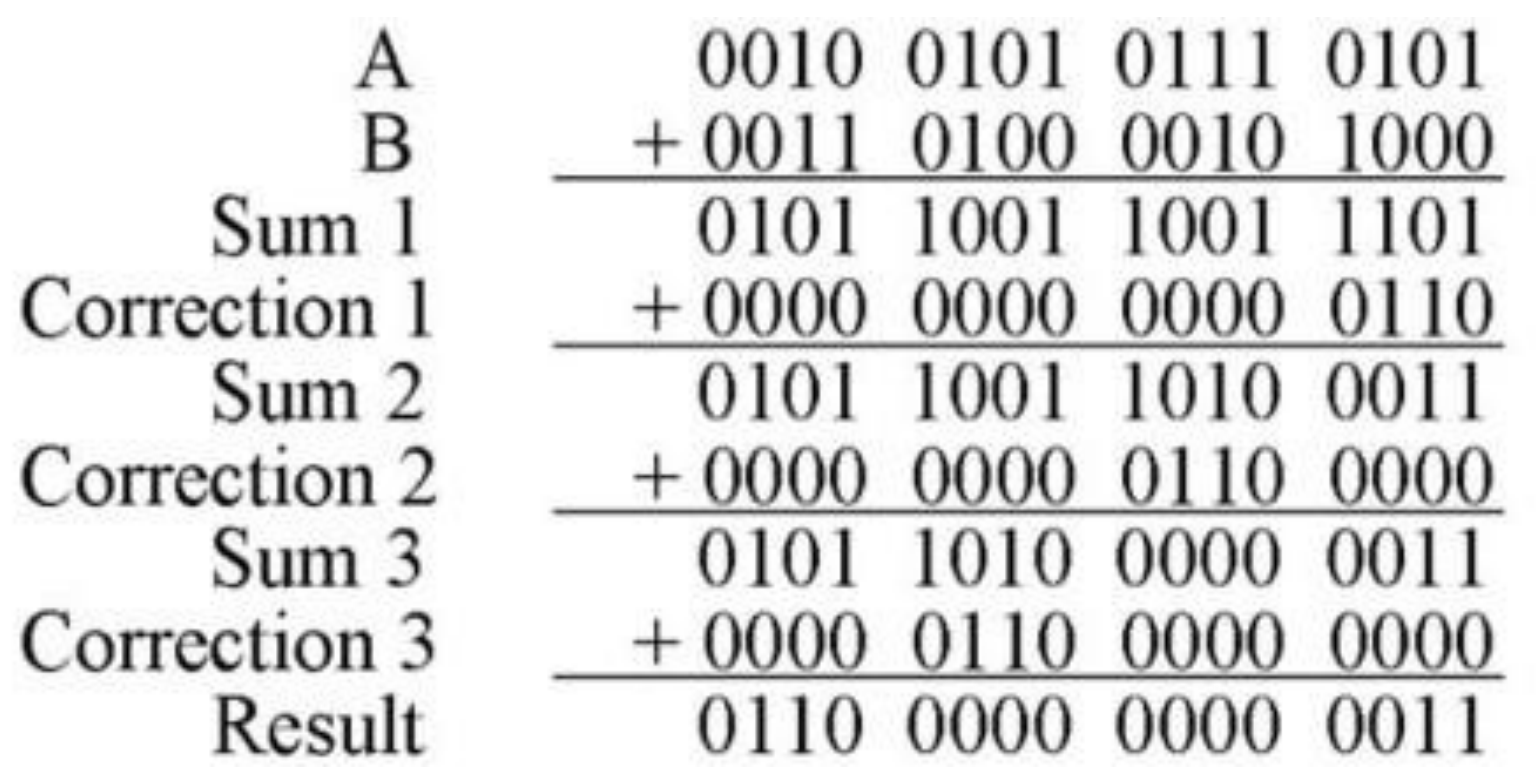

\section{My Architecture (8421-5421 BCD)}

$$
\begin{aligned}
P_{2 n} & =A_{n} \times B_{n}=\sum_{i=0}^{n-1} A_{n} b_{i}(10)^{i} \\
& =\sum_{i=0}^{n-1} A_{n}\left(b 0_{i}+b 1_{i}\right)(10)^{i} \\
& =\sum_{i=0}^{n-1}\left(A_{n}\left(b 0_{i}\right)+A_{n}\left(b 1_{i}\right)\right)(10)^{i} \\
& =\sum_{i=0}^{n-1}\left(P P 0 i_{n+1}+P P 1 i_{n+1}\right)(10)^{i} \\
b_{i} & =5 \times b_{i H}+(-1)^{O P} b_{i L} \\
b_{i H} & b_{i L} \in\{0,1,2\}, O P \in\{0,1\}
\end{aligned}
$$
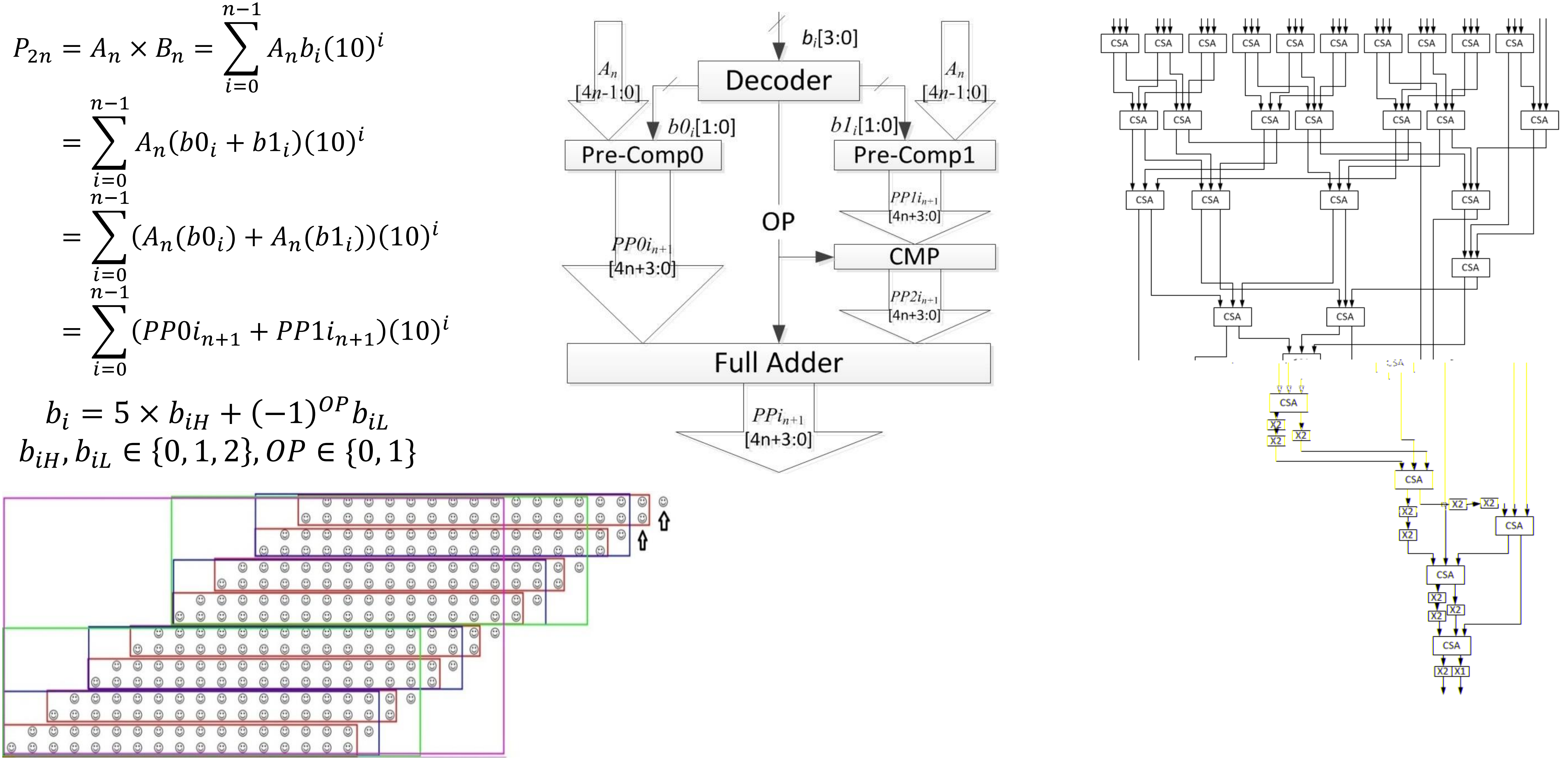

Conclusion:

$>$ Best designs in terms of delay and delay-area product.

$>$ Simplified logic of PPG with 8421-5421 recoding.

8421 CLAs organized as tree structure for PPA

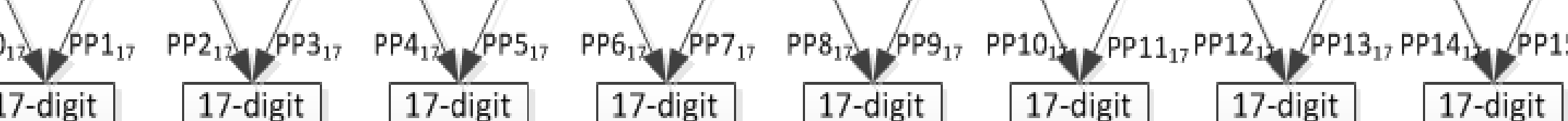

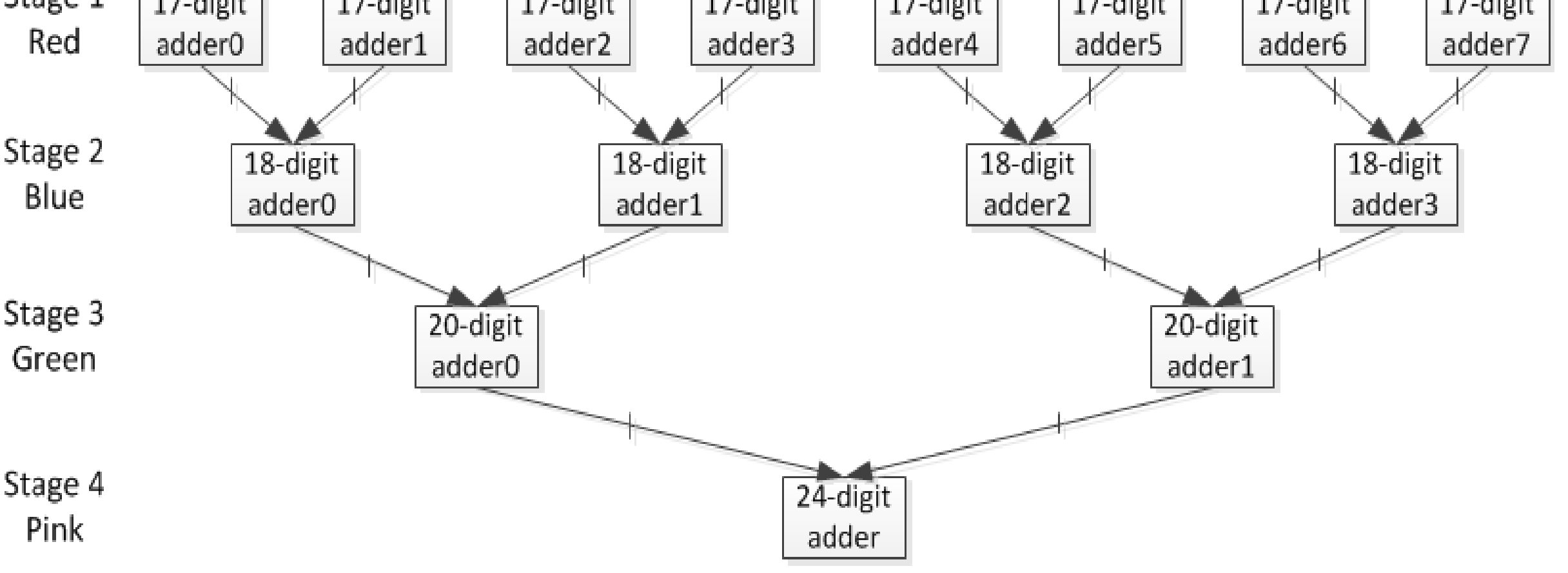

ould be pipelined for higher data throughput and hardware utilization

\section{Evaluation Results}
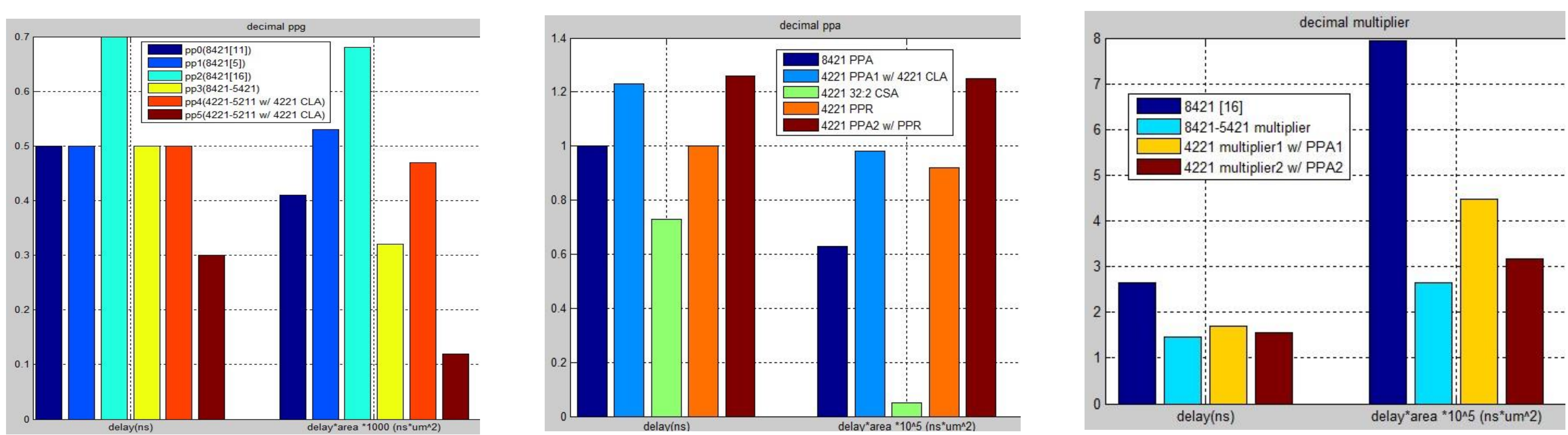